\title{
S-shaped current bistability in a bipolar resonant tunneling diode
}

\author{
O. Kuhn ${ }^{\mathrm{a}, *}$, J. Genoe ${ }^{\mathrm{a}, 1}$, D.K. Maude ${ }^{\mathrm{a}}$, J.-C. Portal ${ }^{\mathrm{a}}$, L. Eaves ${ }^{\mathrm{b}}$, M. Henini ${ }^{\mathrm{b}}$, \\ G. Hill ${ }^{\mathrm{c}}$, M. Pate \\ ${ }^{a}$ Grenoble High Magnetic Field Laboratory, MPI-CNRS, 38042 Grenoble, France \\ ${ }^{\mathrm{b}}$ Deptartment of Physics, University of Nottingham, Nottingham NG7 2RD, UK \\ 'Department of Electrical and Electronic Engineering, University of Sheffield, Sheffield, UK
}

\begin{abstract}
The bipolar tunneling transport through $\mathrm{p}-\mathrm{i}-\mathrm{n}$ double barrier structures has been studied by means of simultaneous electrical transport measurements and electroluminescence spectroscopy. An "inverted" hysteresis loop is observed at the onset of the first electronic resonance in the current-voltage characteristics with an electrical ON/OFF ratio of more than two orders of magnitude. Relating the different branches of the current-voltage characteristic to the space charges accumulated throughout the structure the inverted hysteresis loop is interpreted in terms of an S-shaped current bistability. The S-shaped current bistability is similar to the current driven negative differential resistivity as known for instance from thyristor action. This analogy between the bipolar double barrier structure with alloyed n-type emitter and the thyristor will be briefly discussed. (C) 1998 Elsevier Science B.V. All rights reserved.
\end{abstract}

Keywords: Electroluminescence; Bistability; Resonant tunneling

\section{Introduction}

The incorporation of resonant tunneling structures into $\mathrm{p}-\mathrm{i}-\mathrm{n}$ diodes has led to a new class of optoelectronic quantum devices, namely bipolar resonant tunneling devices. The combination of the features of resonant tunneling, notably negative

\footnotetext{
* Corresponding author. Present address: Dept. of Physics, Chalmers University of Technology, University of Gothenburg, 41296 Gothenburg, Sweden and Niels Bohr Institue, University of Copenhagen, Universitetsparken, 5, 2100 Copenhagen $\varnothing$, Denmark. Fax: + 4631 772-3471; e-mail:kuhn@fy.chalmers.se.

${ }^{1}$ Present address: Vrije Universiteit Brussel, Dept. ETRO, 1050 Brussels, Belgium.
}

differential resistivity, with optical emission from electronic systems of reduced dimensionality, yields light emitters with enhanced functionality. In order to understand their operation numerous questions arise concerning the transport mechanisms that determine, for instance, the space charge accumulation and redistribution in bipolar resonant tunneling devices.

This paper presents a study of bipolar transport through a $\mathrm{p}-\mathrm{i}-\mathrm{n}$ double barrier structure with an alloyed electron emitter. Both electrical transport measurements and electroluminescence spectroscopy are employed for monitoring the electron and hole transport in the device under operational conditions. Section 2 provides a description of the 
sample and the experimental set-up. The data are presented in Section 3. The main result is the hysteresis loop observed in the current-voltage characteristics. This hysteresis loop is discussed in terms of an S-shaped current bistability firstly by regarding the space charges throughout the device under forward bias, and secondly by a comparison to thyristor action.

\section{Experimental}

The sample consists of a double barrier resonant tunneling structure which is embedded into the intrinsic region of a $\mathrm{p}-\mathrm{i}-\mathrm{n}$ diode. The molecular beam epitaxy grown structure is of an asymmetric design, with an alloyed $\mathrm{Al}_{0.09} \mathrm{Ga}_{0.81} \mathrm{As}$ electron emitter, a $5 \mathrm{~nm}$ GaAs quantum well layer in between $6 \mathrm{~nm}$ AlAs barrier layers, and a GaAs p-type contact. A $60 \mathrm{~nm}$ undoped spacer layer separates the doped contact regions from the double barrier structure in order to avoid the diffusion of impurities into the quantum well during growth. A transparent $\mathrm{Al}_{0.33} \mathrm{Ga}_{0.67} \mathrm{As}$ window layer has been incorporated into the top-contact for an improved collection of the light emitted from the double barrier structure. Circular mesas of 50, 100, 200 , and $400 \mu \mathrm{m}$ diameter have been defined by means of conventional photolithographic and wet etching techniques. Ohmic contact to the devices is provided by an annular ring of gold evaporated on top of the mesa. All experiments have been performed at $4.2 \mathrm{~K}$. Current-voltage characteristics have been taken in the four wire configuration. Optical emission from the sample surface is collected via fibre optics, and guided into a $0.64 \mathrm{~m}$ focal length single monochromator. The spectra are recorded with a photomultiplier tube using lock-in amplification.

\section{Results}

Fig. 1 shows the current-voltage characteristics of the bipolar double barrier structure with a $5.2 \mathrm{~nm}$ quantum well. The onset of the current occurs at a voltage of $1.53 \mathrm{~V}$. Two current resonances related to resonant injection into the first

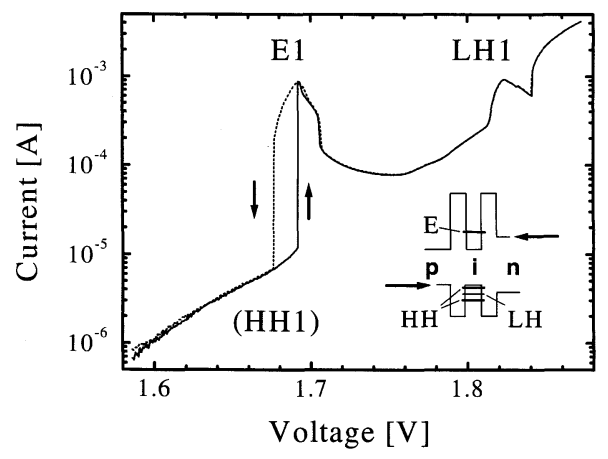

Fig. 1. Current-voltage characteristics of the bipolar double barrier structure with alloyed n-type emitter taken at $4.2 \mathrm{~K}$. Both up-sweep (solid line) and down-sweep (broken line) are shown. Note the "inverted" hysteresis at $1.7 \mathrm{~V}$. The labels HH1, $\mathrm{E} 1$, and LH1 refer to the heavy hole, electron and light hole resonances, respectively. (Inset) Schematic of the $\mathrm{p}-\mathrm{i}-\mathrm{n}$ GaAs/AlAs/AlGaAs double barrier structure under flat band conditions. Only the lowest states in the quantum well are shown. Electron and heavy hole states are drawn as thick lines, light hole states as thin lines. The arrows indicate charge carrier injection.

electronic (E1) and the first light hole level (LH1) in the quantum well occur at 1.69 and at $1.83 \mathrm{~V}$, respectively. Above $1.84 \mathrm{~V}$ the current drastically increases as electron transport via AlAs X-states dominates the current. The most remarkable aspect about the current-voltage characteristic is the hysteresis loop observed at the E1-resonance. This hysteresis loop occurs on the rising part of the resonance and is inverted as compared to what is usually observed for current bistabilities in resonant tunnelling structures. The peak current in the high-current state and the current in the corresponding low-current state differ by more than two orders of magnitude.

Typical electroluminescence spectra taken at various applied bias voltages around $1.7 \mathrm{~V}$ are shown in Fig. 2. Light emission is seen from the quantum well as well as from the bulk contact regions. The quantum well luminescence intensity occurs at $1.65 \mathrm{eV}$. The luminescence between 1.55 and $1.63 \mathrm{eV}$ is attributed to recombination in the n-type $\mathrm{Al}_{x} \mathrm{Ga}_{1-x} \mathrm{As}$ contact. The broad luminescence peak at around $1.5 \mathrm{eV}$ originates from the p-type GaAs contact.

The relative intensities of the luminescence peaks strongly depend on the applied bias. Fig. 3 depicts 


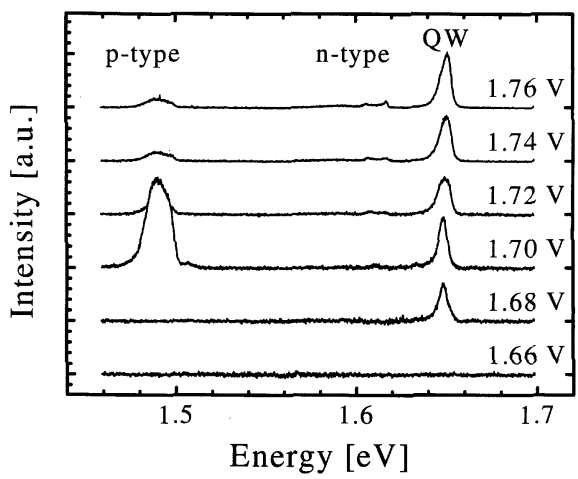

Fig. 2. Typical electroluminescence spectra taken at $4.2 \mathrm{~K}$ under various forward bias voltages. Note the sequence in which the different features appear in the spectra as a function of bias. The quantum well emission (QW) already appears in the $1.68 \mathrm{~V}$ spectrum, before the p-type GaAs luminescence (p-type) and at higher voltages the n-type AlGaAs luminescence (n-type) are seen.

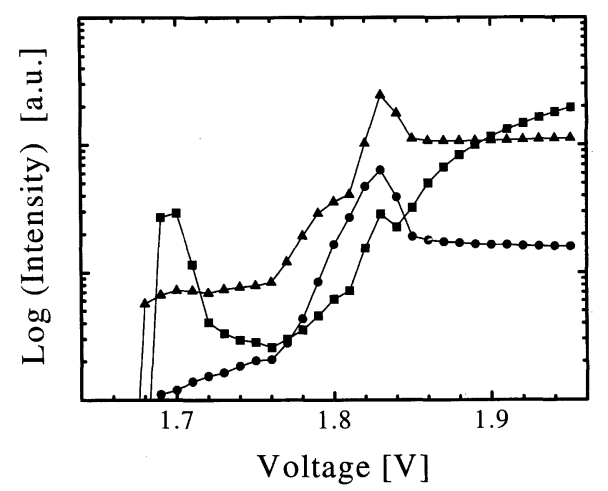

Fig. 3. Voltage dependence of the electroluminescence intensity $(T=4.2 \mathrm{~K})$. The p-type GaAs luminescence is marked by squares, the n-type AlGaAs luminescence by dots, and the quantum well luminescence by triangles.

the evolution with applied bias voltage of the electroluminescence intensities integrated over the different spectral ranges associated with recombination in the quantum well, in the p-type GaAs, and in the n-type AlGaAs bulk contacts. A correlation with the current-voltage characteristic is evident. The first electroluminescence line to appear is that of the quantum well recombination. It exhibits a step-like onset at $1.68 \mathrm{~V}$ and remains almost constant up to $1.76 \mathrm{~V}$. Above $1.76 \mathrm{~V}$ the intensity of the quantum well emission progressively increases until at $1.83 \mathrm{~V}$ it forms a pronounced resonance with a peak to valley ratio of more than $2: 1$. Almost simultaneously with the quantum well luminescence, the p-type GaAs contact luminescence switches on at $1.69 \mathrm{~V}$, and a marked resonance with its peak at $1.7 \mathrm{~V}$ is observed. The resonance essentially follows the current-voltage characteristic. It has a peak to valley ratio of $10: 1$, comparable to what is observed for the current. The electroluminescence intensity in the p-type GaAs contact is at its minimum at $1.76 \mathrm{~V}$. Above, the p-type luminescence constantly increases with a strong gain in slope at $1.82 \mathrm{~V}$. Only a small feature is present at $1.83 \mathrm{~V}$. The n-type AlGaAs contact luminescence, on the other hand, displays a clear peak at this voltage. Note that a significant n-contact luminescence is only observed for voltages above $1.76 \mathrm{~V}$.

A short discussion of the contact luminescence intensities illustrates the assignment of the different features in the current-voltage characteristic to conduction band or to valence band transport processes. First, we note that the p-contact luminescence intensity, closely related to the electronic transport, peaks at around $1.7 \mathrm{~V}$. The corresponding peak in the total current is therefore obviously due to a conduction band transport resonance, namely the resonant injection of electrons into the $\mathrm{E}_{1}$-subband in the quantum well. The strong onset in p-contact luminescence intensity at around $1.82 \mathrm{~V}$ coincides with a strong increase in current. This confirms that the strong current onset at voltages above $1.82 \mathrm{~V}$ is due to the opening up of the $\Gamma-\mathrm{X}$ transport channel. The voltage dependence of the n-type AlGaAs contact luminescence identifies the transport process underlying the resonance at $1.83 \mathrm{~V}$ as being related to the tunnelling of holes, evidently the resonant injection of holes into the $\mathrm{LH}_{1}$-subband of the quantum well. No resonance is observed in the n-contact luminescence intensity for resonant injection of holes into the lowest valence band state of the quantum well, the $\mathrm{HH}_{1}$ subband, because at the corresponding voltages holes injected from the p-type GaAs contact are blocked in the quantum well by the alloyed ncontact. 


\section{Space charge}

The most intriguing feature of the bipolar double barrier structure with alloyed n-type emitter is the "inverted" hysteresis loop observed in the current-voltage characteristic. The device switches from the low current state to the high current state at higher voltages than from the high current state to the low current state. Interpreting the inverted hysteresis loop in terms of a so-called S-shaped current bistability [1] the different branches of the current-voltage characteristic may be related to the space charges accumulated throughout the structure. Fig. 4 shows schematically a current-voltage characteristic exhibiting an S-shaped bistability. When applying a forward bias beyond flat band conditions (0), electron and hole charges are built up next to the electron and hole emitter barriers respectively (1). The electric field across the double barrier structure is uniform until hole injection into the first quantum well heavy hole level becomes
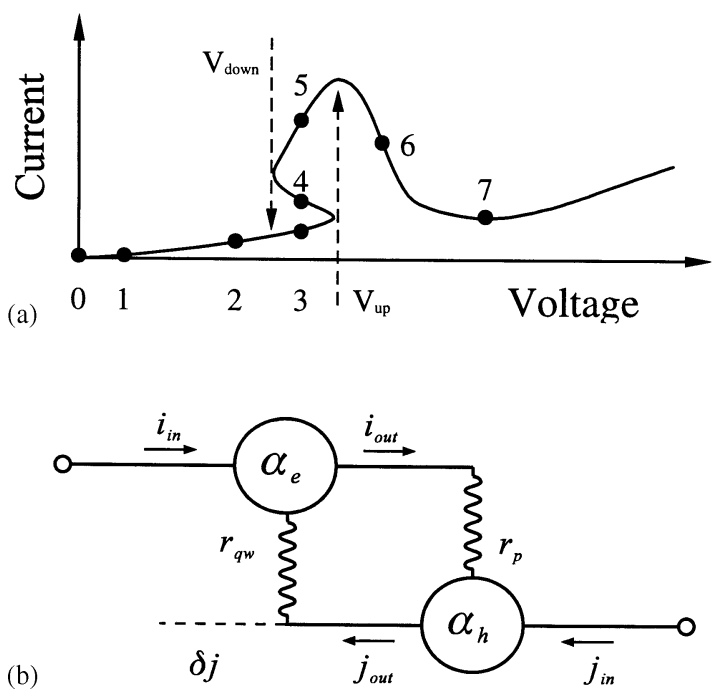

Fig. 4. (a) Schematic of the current density versus electric field characteristics for a bipolar double barrier structure exhibiting a region of current controlled negative differential resistivity. The S-shaped characteristic leads to a hysteresis loop as shown when measuring the current for an externally controlled electric field across the device. (b) Two-node representation of the bipolar double barrier structure with alloyed n-contact in analogy to the two-transistor model of a general thyristor under forward bias. possible. Positive charges accumulate in the quantum well leading to a larger electric field across the electron emitter barrier than across the hole emitter barrier (2). Any further increase of bias now mainly increases the electric field across the electron emitter barrier (3). The maximum hole charge in the well is achieved at the turning point $V_{\text {up. }}$. However, in the whole electric field region between $V_{\text {down }}$ and $V_{\text {up }}$ three different charge distributions and thus electric field configurations are stable for the same drop of electrostatic potential across the double barrier structure: a state with an excess of hole charge (3), a state with an excess of electron charge (5), and an intermediate state where the net charges in the well cancel (4). The two former states $(3,5)$ exhibit positive differential resistivity, whereas the intermediate one is characterised by negative differential resistivity (4). At electric fields above $V_{\text {up }}$ the quantum well now negatively charged, discharges gradually (6) until finally a situation of uniform electric field is recovered (7). When measuring the current as a function of externally set bias voltage, i.e. along a vertical load-line, jumps occur at the points $V_{\text {up }}$ and $V_{\text {down }}$ of vanishing $\mathrm{d} V / \mathrm{d} I$.

Switching between the two current states with positive differential resistivity, (3) and (5), is controlled by the charges present in the quantum well. In other words, which of these states is favoured depends on whether electron or hole supply is more efficient, and on how much charge of either kind is already present in the well. When entering the bistability regime coming from low voltages the quantum well is positively charged (the $\mathrm{HH}_{1}$-resonance occurs at lower voltages than the $\mathrm{E}_{1}$ resonance). The positive charge in the quantum well acts to keep the device on resonant hole injection and the low current state persists. In order to switch the device to the high current state an additional voltage needs to be applied that drives the structure beyond resonant hole injection, onto resonant electron injection so that the net charge in the quantum well can become negative. The situation is reversed when entering the bistability regime from high voltages. In this case the high current state with a negative net charge persists. The device switches to the low current state as soon as the applied bias voltage is reduced such that the net charge in the quantum well becomes positive. 


\section{Analogy to the thyristor}

For illustrating the working principle of the bipolar resonant tunneling diode with alloyed n-emitter an analogy to thyristor action can be invoked. The S-shaped current bistability resembles the current driven negative differential resistivity of thyristors. The three main branches of a thyristor current-voltage characteristic under forward bias conditions, the forward blocking ("OFF") state, the negative differential resistance region, and the forward conducting ("ON") state correspond to the points (3), (4), and (5) in Fig. 4, respectively. A model that is commonly used for discussing the basic characteristics of a thyristor is the two-transistor analogue. In this model, the thyristor, a four-layer $\mathrm{p}-\mathrm{n}-\mathrm{p}-\mathrm{n}$ device, is represented by a pnp-transistor and an npn-transistor with the base electrodes connected to the respective other transistor [2]. A similar scheme can be set up for analysing the bistable behaviour in the bipolar double barrier structure with alloyed n-emitter (Fig. 4b). In this scheme, the transistors are replaced by a three-terminal node. The pnp-transistor is transposed into a node for electrons, and the npn-transistor into a node for holes. The emitter current is identified with the charge carrier injection, the collector current with charge carrier escape, and the base current with recombination. The nodes are characterised by a transmission factor $\alpha$ that relates the injection current $i_{\text {in }}$ both to the charge carrier escape $i_{\text {out }}=\alpha i_{\text {in }}$, and to the recombination $r=(1-\alpha) i_{\text {in }}$. A three-terminal node thus describes an electroluminescent layer. In the voltage regime of the current bistability two regions of the structure are active, the quantum well and the hole accumulation layer in the p-type GaAs contact next to the AlAs barrier. The electron node, with a transmission factor $\alpha_{\mathrm{e}}$, represents the quantum well. It is supplied via (resonant) tunneling injection $\left(i_{\text {in }}\right)$ and drained by tunneling escape of electrons $\left(i_{\text {out }}\right)$. The hole node, with a transmission factor $\alpha_{\mathrm{h}}$, symbolises the hole accumulation layer. This node is supplied by charge transfer from the heavily p-type doped GaAs region $\left(j_{\text {in }}\right)$. Note that in this scheme the quantum well acts as a collector to the hole node $\left(j_{\text {out }}\right)$.
Recombination in the AlGaAs n-contact is small, because of an almost complete blocking of the holes in the quantum well. Only a small current $\delta j$ of thermally activated holes flows into the ncontact. Virtually all holes entering the quantum well are annihilated by recombination. Equating the recombination rate of electrons in the quantum well with the hole current, $r_{\mathrm{qw}}=j_{\text {out }}-\delta j$, we find an expression for the total current $I$ through the device:

$I=j_{\text {in }}=\delta j \frac{\alpha_{\mathrm{e}}}{\alpha_{\mathrm{e}}+\alpha_{\mathrm{h}}-1}$.

Eq. (1) is valid for low electron injection, where the number of electrons limits and thus modulates the recombination in the quantum well. This situation corresponds to the low current state, or in the terminology of the thyristor characteristics to the forward blocking. As $\delta j$ is a small quantity the total current is also small unless the sum of $\alpha_{\mathrm{e}}$ and $\alpha_{\mathrm{h}}$ approaches unity. When the right-hand side of Eq. (1) diverges the device switches to the high current, or forward conducting state.

\section{Conclusion}

The transport through a bipolar GaAs/AlAs double barrier structure with a GaAs p-type contact and an $\mathrm{Al}_{0.09} \mathrm{Ga}_{0.91} \mathrm{As}$-type contact has been studied. The asymmetric contact design is found to introduce a hysteresis in the current when measured as a function of externally applied bias voltage around the first electron resonance. Two aspects of this hysteresis loop are remarkable. Firstly, the current differs by more than two orders of magnitude between the high current state and the low current state. Secondly, the hysteresis loop is inverted as compared to what is usually observed in resonant tunnelling devices. An S-shaped bistability is proposed as the underlying, intrinsic current-voltage characteristic. The S-shaped bistable behaviour is intimately related to the bipolar character of the transport. The device characteristics has therefore been discussed with regard to the space charges throughout the device under 
operation, and from a second point of view by comparison to thyristor action.

\section{Acknowledgements}

Part of this work has been funded by the European Community under ESPRIT project No. 7193 "PARTNERS". J.G. and O.K. would like to acknowledge financial support by the European
Community. O.K. would also like to thank the Knut and Alice Wallenberg Stiftelse, Sweden, for financial support.

\section{References}

[1] See, e.g., Wacker, Schöll, J. Appl. Phys. 78 (1995) 7352.

[2] S.M. Sze, Physics of Semiconductor Devices, 2nd ed., Wiley, New York, 1981, p. 190. 